\title{
CONTRIBUTIONS

\section{Description of a new Limnophora species from Bulgaria (Diptera: Muscidae)}

With 4 figures

EBERHARD ZIELKE 1

${ }^{1}$ Institute of Biodiversity and Ecosystem Research, Bulgarian Academy of Sciences, 1 Tsar Osvoboditel Blvd, 1000 Sofia, Bulgaria. - eo.zielke@abv.bg

Published on 2017-12-08

DOI: $10.21248 /$ contrib.entomol.67.2.319-323'

\section{Abstract}

Limnophora ljubomirovi spec. nov. with slightly curved wing-vein $\mathrm{M}_{1}$ and long plumose hairs of arista is described from Bulgaria and compared with similar species of the genus.

\section{Nomenclatural acts}

Limnophora ljubomirovi spec. nov. - iurn:lsid:zoobank.org:act:BC851 ACE-FCBA-482F-800D-70177499A33A

\section{Key words}

Limnophora ljubomirovi spec. nov., Bulgaria

\section{Zusammenfassung}

Limnophora ljubomirovi spec. nov. mit einer leicht nach vorne gebogenen Flügelader $\mathrm{M}_{1}$ und langen Aristahaaren wird als neue Art von Bulgarien beschrieben und mit bekannten ähnlichen Arten verglichen.

\section{Introduction}

A male of the genus Limnophora Robineau-Desvoidy, 1830 has been collected recently by sweeping shadowed vegetation in the river bed, directly at the water side of Vurbitsa river near to Momchilgrad in the southern part of the Eastern Rhodopes. The specimen is characterized, beside other markings, by the longest aristal hairs as long as width of postpedicel and by a slightly curved wing-vein $\mathrm{M}_{1}$. The species is described as Limnophora ljubomirovi spec. nov.

\section{Material and Methods}

Keys to the Muscidae of the Palaearctic Region (Hennig, 1964) and to the Muscidae of Central Europe (Gregor et al. 2002 and 2016) have been used for identification. External morphological features were examined using a ZEISS Stemi 2000-C stereomicroscope, for illustrations an AxioCam ERc5s camera and for further processing Helicon Focus 6 and Adobe Photoshop CS2 have been applied. Standard terminologies as applied by GREGor et al. (2016) in the Manual of Central European Muscidae 
(Diptera) are also used for the description. Body length was measured in millimeters ( $\mathrm{mm})$.

After finalizing this description of the new species the right front and mid leg of the holotype have been removed and preserved in $90 \%$ ethanol to allow future investigations on DNA sequences of this species. The holotype and the vial (Nr. D 48) with the preserved legs are deposited in the entomological collection of the Institute of Biodiversity and Ecosystem Research, Sofia.

\section{Results}

Limnophora ljubomirovi spec. nov.

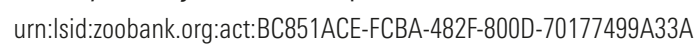

Material examined: Male holotype, Bulgaria, Vurbitsa river bed, Momchilgrad, 41 $32^{\prime} 34^{\prime \prime} \mathrm{N} ; 25^{\circ} 23^{\prime} 23^{\prime \prime E}, 239 \mathrm{~m}$, 15.8.2016, leg. E. Zielke.

Description: Head. Ground-colour black. Eyes bare. Frons (fig. 1) slightly dilating from vertex to lunula, at middle 0.47 times as broad as width of head. Frontoorbital plate at level of anterior ocellus about as broad as twice the diameter of anterior ocellus, at anterior margin broader than width of postpedicel. Frontal vitta dilating from top to middle and then getting slightly narrower, frontal triangle visible as a whole only at certain point of view reaching almost anterior margin. Parafacial about evenly broad all over the length, at middle as broad as two third of width of postpedicel. In profile antennae inserted nearly at middle of head, upper mouth margin about in line with profrons. Genal depth below lowest eye margin equal to width of postpedicel. In anterodorsal view fronto-orbital plates greyish-white with a conspicuously brown patch at about middle, extending from second to most posterior frontal seta. Frontal vitta and lower part of frontal triangle, depending on angle of view, dark to almost velvety black, upper part of frontal triangle and the ocellar tubercle greyish white. Face and parafacial from anterior point of view white and shining white respectively; gena and post-occipital surface uniformly greyish at lateral view. All antennal segments black, postpedicel three times as long as wide, tip dorsally with a rounded edge. Arista about twice as long as length of postpedicel, longest aristal hairs about as long as width of postpedicel. Ocellar setae strong, about as long as inner verticals, outer verticals slightly longer than half length of inner verticals and distinctly longer than adjacent postocular setae. Fronto-orbital plate at level of anterior ocellus and above with two distinct reclinate orbital setae (anterior ones are missing but scars are very distinct), one very strong inclinate frontal seta at the lower margin and distinctly above, at about the middle third of frons in the area of the brown patch, two or three weaker frontal setae, one or two short interstitial hairs between the lowest and the upper frontal setae, lower part of fronto-orbital plate, in particular the brown patch with several short setulae outside the frontal setae. Parafacial bare. Vibrissal setae strong and more than twice as long as the surrounding peristomal setae. Lower margin of gena, post-genal and post-occipital surfaces covered with dark hairs. Proboscis shining black; palpus black and slender.

Thorax: Ground-colour grey. Scutum from dorsal point of view (fig. 2) predominantly greyish; presutural part of scutum with a pair of broad brown submedian stripes reaching from neck to posterior presutural dorsocentral seta, each stripe widens in posterior half to an almost square patch which lies about between the presutural dorsocentrals and the posthumeral and presutural setae, at suture two narrow but somewhat broadened brown presutural patches; postsuturally a somewhat shining dark brown transverse band, extending between the wing bases and from suture to about the insertion of second postsutural dorsocentral seta. Basis of brown transverse band, directly at suture with two narrow, crescent-shaped grey patches each one about as broad as distance between the row of postsutural dorsocentrals and the intra-alar setae. A brown crescent like median spot located on scutum directly at scutellar suture. Scutellum with transverse shining dark brown band covering about the basal half of dorsal and lateral surfaces, posterior half dorsally and laterally uniformly grey, ventral surface predominantly dark. Greater ampulla and adjacent area brown, pleura all grey. Anterior and posterior spiracle whitish. Scutum and pleura with some dark setulose groundhairs, hairs of pleura distinctly shorter than those of scutum. Thorax with $0+1$ acrostichal setae, the presutural setulae in about four irregular rows; dorsocentral setae $2+3$, prescutellar one much longer than anterior one; notopleuron with 2 setae but without additional hairs; 2 intra-alar setae. Scutellum with 1 long apical and 1 long lateral seta, preapical and basal setae weak and only slightly longer than surrounding hair, lateral and ventral surfaces bare. Prosternum laterally with black setulae. Proepimeral area, anepimeron, katepimeron and meron bare. Katepisternals $1+2$, the posterior upper one about three times as long as the two other ones. Anepisternum at posterior margin with about 4 stronger setae and few interstitial hairs.

Wing: Membrane hyaline. Basicosta and tegula dark brown; veins at basis yellowish and more distally brown. Costal spine not significantly longer than surrounding costal bristles. Veins bare, radial node dorsally and ventrally with few long setulae, the longest ones about as long as half the length of crossvein r-m or even longer. Crossvein $\mathrm{r}-\mathrm{m}$ below the point where vein $\mathrm{R}_{1}$ enters costa, distal crossvein $\mathrm{dm}-\mathrm{cu}$ sinuous, barely oblique. Vein $M_{1}$ slightly curved forward before wing margin (fig. 3). Calypters whitish transparent with white margin and fringe, lower calypter distinctly longer than upper calypter. Haltere predominantly white with a very weak yellowish shade. 
Legs: Ground-colour dark, somewhat greyish. Pulvilli about half as long as claws, which are barely half as long as corresponding tarsomere 5. Hind coxa bare on posterior surface. Fore femur with complete rows of posterodorsal, posterior and posteroventral setae, all setae barely as long as depth of femur; fore tibia without posterior seta. Mid femur at basal half with row of anterior setae, the longest one at middle of femur, barely much longer than half width of femur, preapically two strong posterior setae; mid tibia with two posterior setae longer than diameter of tibia. Hind femur with complete row of anterodorsals and at apical fourth with about three anteroventrals, all setae not longer than depth of femur; hind tibia with one anteroventral and one anterodorsal seta about as long as diameter of tibia.

Abdomen: Ground-colour grey (fig. 4). Posterior half of tergite $1+2$ with two paramedian brown patches of almost round shape reaching posterior margin; tergite 3 from anterior to posterior margin with a pair of broad paramedian longitudinal stripes which extend in posterior half laterally forming an L-shaped marking; tergite 4 in posterior half with two paramedian brown patches reaching the posterior margin; tergite 5 only with a median longitudinal stripe not reaching the margins. Tergite 1+2 laterally with several longer setae, tergites 3 and 4 laterally with some longer marginal setae; tergite 5 with a complete row of long discal setae and with short and weak marginals. Sternite 1 bare; sternites 2 to 5 without conspicuous setae, sternite 5 with almost completely straight posterior margin (fig. 5).

Male genitalia: Hypopygium barely pronounced. The species is clearly distinguished by morphological characters from similar species. The determination does not depend on characters of male terminalia. To avoid inflicting damage on the only hitherto available specimen of this species it has been refrained from extracting the genitalia.

Measurements: Length of body about $5.5 \mathrm{~mm}$; length of wing about $5 \mathrm{~mm}$.

Female not known.

Etymology: It is a pleasure to name this species after Toshko Ljubomirov, Ph.D., Associate Professor and curator of the scientific zoological collection of the Institute of Biodiversity and Ecosystem Research, Sofia. I want to express herewith my gratitude for his continuous help and active support when field trips had been planned and conducted, to collect muscids in different localities and biotopes of Bulgaria. The species name ljubomirovi is a noun in the genitive case.

Diagnosis: Limnophora ljubomirovi is in particular characterized by a plumose arista, conspicuous brown patch at the middle of the greyish-white frontoorbital plate and the slightly but clearly curved wing-vein $M_{1}$ before margin of wing. These and other taxonomic markings lead in the key to Limnophora species (Hennig, 1964) to couplet $22(23)$ with Limnophora exigua (WIEDEMANN, 1830) (= Limnophora plumiseta SteIn, 1903). This species is not recorded yet

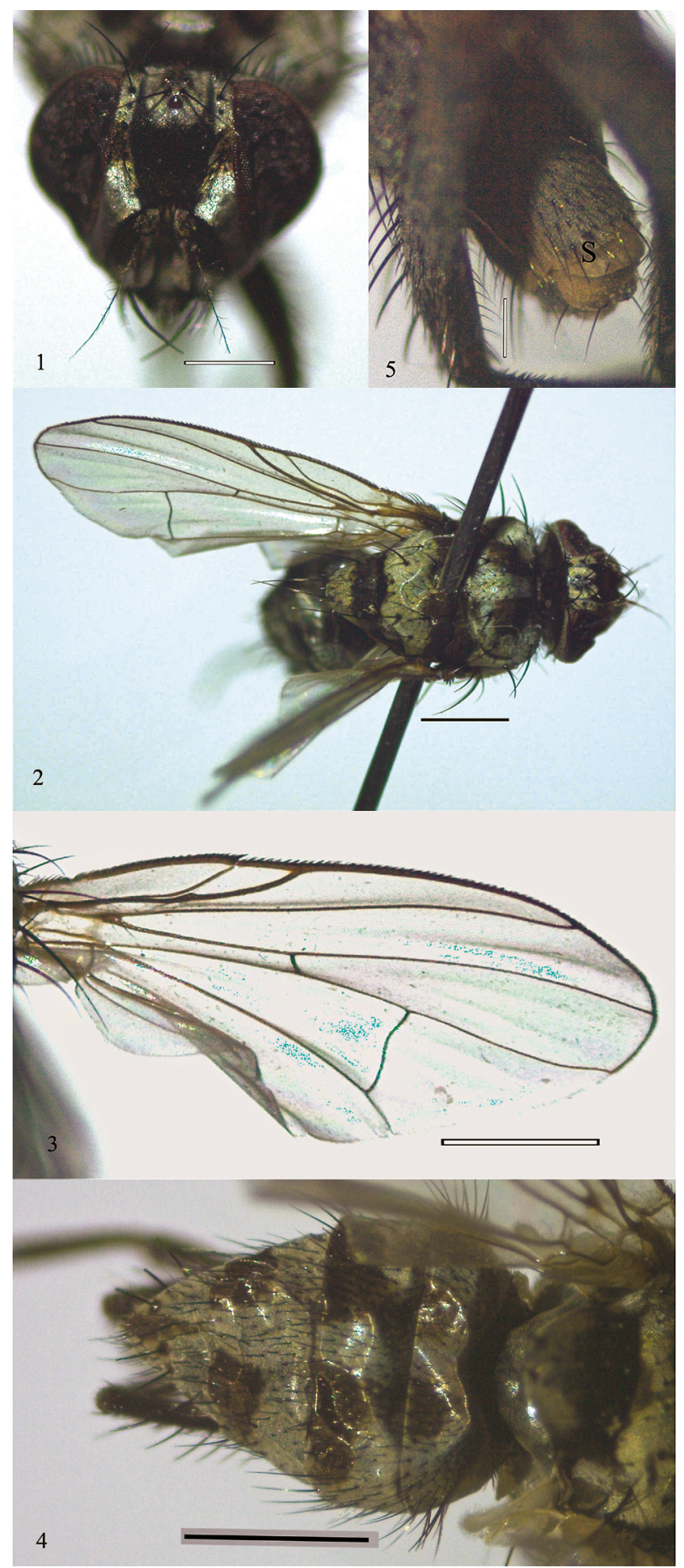

Limnophora ljubomirovi spec. nov., male holotype. (Fig. 1: frons viewed from anterodorsal (bar $=0.5 \mathrm{~mm}$ ). Fig. 2 : dorsal view of scutum (bar = $1 \mathrm{~mm}$ ). Fig. 3 : dorsal view of right wing with vein $\mathrm{M} 1$ slightly curved forward to vein $\mathrm{R}_{4+5}($ bar $=1 \mathrm{~mm})$. Fig. 4: male holotype, dorsal view of abdomen $($ bar $=1 \mathrm{~mm})$. The anterior half of tergite $1+2$ is grey and not as dark, as it appears on the photo. Fig. 5: ventral view of sternite 5 (S) (white bar $=0.2 \mathrm{~mm})$ ). 
from Europe, but known from Asia, North Africa and Afrotropical Region. Both species have in common aristal hairs as long as or longer than half the width of postpedicel. Additionally to other differences such as colour of frons and markings of thorax and abdomen these species are clearly distinguished by $2+4$ dorsocentral setae and parallel running wing-veins $\mathrm{R}_{4+5}$ and $\mathrm{M}_{1}$ in L. exigua and $2+3$ dorsocentrals and the apically curved vein M1 in L. ljubomirovi. Using the newly published keys by Gregor et al. (2016) which primarily consider Muscidae from Central Europe, the male of L. ljubomirovi leads to couplet 8 with Limnophora triangula (FALlén, 1825). L. triangula is characterized by short hairs on the arista, more or less strongly developed longitudinal stripes on scutum, mid tibia with only one posterior seta and parallel running wing veins $R_{4+5}$ and $M_{1}$, whereas in L. ljubomirovi the aristal hairs are about as long as width of postpedicel, the markings of scutum and scutellum are transverse, mid tibia has two posterior setae and the mentioned wing veins are apically not parallel. Moreover, there are also differences between the two species regarding colour of head, thorax and abdomen.

The specific pattern of thorax and abdomen of the L. ljubomirovi male lets one think at the first glance of the markings known from females of Limnophora tigrina Am STEIn, 1860. However, the male of L. ljubomirovi has a postsutural brown transverse band reaching from wing base to wing base, whereas the females of L. tigrina are marked with two distinctly smaller brown patches, which are very similar to the presutural ones.

A slightly curved vein $M_{1}$ is also known from species of the genus Neolimnophora SCHNABL which is closely related to the genus Limnophora and which is separated from Limnophora by ventrally and dorsally bare radial nodes. L. ljubomirovi has well developed setae on both sides of the radial node and can also be differentiated from the two known Neolimnophora species by its long aristal hairs, $2+3$ dorsocentral setae and different body colour and pattern.

\section{Discussion}

The estimated number of worldwide known species of the genus Limnophora RoBINEAU-Desvoidy varies between 230 (XuE et al., 2012) and 350 species (Pont et al., 2011). Limnophora is well represented in the Palaearctic region and several species have been in recent years subject to studies regarding biology, distribution and taxonomy (e.g. Ivkovic \& Pont 2015 and 2016, Pont \& Ivkovic 2013, Pont et al. 2011, Werner \& Pont $2006 \mathrm{a}+\mathrm{b}$, Xue et al. 2012). In 2013 one new species of the genus, Limnophora croatica, has been described from Croatia by PONT \& Ivkovic. This species has not been considered by HeNNIG's revision (1964) published almost 50 years ago, and it is also not included in the recently published manual by Gregor et al. (2016) with the focus on Central European Muscidae. L. croatica and L. ljubomirovi, the only two new Limnophora species described in recent years from the European part of the Palaearctic Region, are, however, very different. L. croatica is a member of the Limnophora riparia - group which is characterized by having the antennae inserted distinctly above middle of head whereas in L. ljubomirovi antennae are positioned at about the middle of the head. Additionally L. croatica is marked by dark brown pruinose frontoorbital plates, short pubescent arista, predominantly dark thorax and abdomen, without conspicuous pattern on scutum and parallel running wing-veins $\mathrm{R}_{4+5}$ and $\mathrm{M}_{1}$, whereas L. ljubomirovi has greyish-white frontoorbital plates marked with a brown patch at middle, long aristal hairs, uniformly greyish thorax and abdomen with conspicuous transverse markings on scutum and apically a slightly curved vein $M_{1}$.

The number of Limnophora-species recorded from Bulgaria (Pont, 2013) is raised now with L. ljubomirovi to 12 species.

\section{Acknowledgements}

I am very grateful to Toshko Ljubomirov $\mathrm{Ph}$. D., Associate Professor and curator of the scientific zoological collection of the Institute of Biodiversity and Ecosystem Research, Sofia for giving me generous access to the Diptera collection and for providing all facilities needed for the examination of the material. I also have to thank two anonymous reviewers for valuable comments and helpful suggestions for the improvement of this paper.

\section{References}

Gregor, F.; Rozkošny, R.; BARták, M. \& VAŇHara, J. 2002: The Muscidae (Diptera) of Central Europe. Folia Facultatis Scientiarum Naturalium Universitatis Masarykianae Brunensis, Biologia 107: 1-280.

Gregor, F.; Rozkošny, R.; Barták, M. \& VaŇhara, J. 2016: Manual of Central European Muscidae (Diptera). - Zoologica 162: 1-220.

Hennig, W. 1964: Muscidae.: 1-1110. - In: Lindner, E. (ed): Die Fliegen der palaearktischen Region. 63b E. Schweizerbart'sche Verlagsbuchhandlung, Stuttgart.

Ivković, M. \& Pont, A. C. 2015: New records of Muscidae (Diptera) from Mediterranean countries. _ ZooKeys (496): 131-144. - DOI 110.3897/zookeys.496.9445.

Ivкović, M. \& PonT, A. C. 2016 : Long-term emergence patterns of Limnophora species (Diptera, Muscidae) in specific karst habitats; tufa barriers. - Limnologica 61: 29-35. 
Pont, A. C. 2013: Fauna Europaea: Muscidae. - In: PApe, T. \& Beuk, P. (eds): Fauna Europaea: Diptera Brachycera. - Fauna Europaea, version 2.6.2. - http://, iwww.fauna-eur.org. 2013

Pont, A. C. \& Ivković, M. 2013: The hunter flies of Croatia (Diptera: Muscidae: genus Limnophora Robineau-Desvoidy). - Journal of Natural History 47(15-16): 1069-1082.

Werner, D. \& Pont, A. C. 2006a: The feeding and reproductive behaviour of the Limnophorini (Diptera: Muscidae). - Studia dipterologica. Supplement 14: 79-114.
Werner, D. \& Pont, A. C. 2006b: New results on Diptera predators in the blackfly plague areas of Central Europe and the Caucasus. - Acta entomologica Serbica. Supplement: 131-140. - Thttp://www.eds.org.' rs/AES/Vol11Suppl/16.pdé, [accessed $20 \overline{1} \overline{7} / 0 \overline{8} / \overline{2} \overline{9}]$.

XuE, W.-QQ; $\bar{B}$ AI, S.-C. \& DONG, W.-X. 2012: A study of the genus Limnophora Robineau-Desvoidy (Diptera: Muscidae), with descriptions of six new species from China. - Journal of Insect Science 12(1): 1-20 - DOI $10.1673 / 031.012 .1701$ 


\section{Besprechung / Review}

Hofmann, A. F. \& Tremewan, W. G. 2017: The natural history of burnet moths (Zygaena FABRICIUs, 1775) (Lepidoptera: Zygaenidae), part 1. - Proceedings of the Museum Witt, Munich, Vilnius: 631 pp. ISBN 978-3-940732-32-3.

For all friends of burnet moths, this year is an excellent year because the first volume of THE Hofmann and Tremewan was published. This work which has been elaborated during decades of intensive work with Zygaeninae of the two authors is compiling so much information on its 632 pages that everyone getting the book in hands (even if only marginally interested in the topic) will not stop reading and will become a great friend of these lovely little and fascinating creatures, burnet moths! While Naumann and colleagues already published an excellent and comprehensive book on the Zygaenidae of the western Palaearctic in 1999, this book now being published opens a series of three volumes, which will be even much more in depth than all previous publications making the natural history of the genus Zygaena one of the best studied of all insect groups. All aspects in this book are described in very detail and the text is always accompanied by many tables, maps, pictures and illustrations of very high clarity and quality.

This first volume on the natural history of burnet moths reviewed here is structured in eight chapters. The first chapter is briefly introducing into the subject and is giving a very short summary over all three volumes. The second chapter provides an overview on the origin, phylogeny and systematics of the genus Zygaena also including the other representatives of the Zygaeninae and a checklist of all Zygaena species including the structuring in subgenera and lower taxonomic groups. The distributions of all these groups are depicted on maps and the phylogeographic implications are discussed very competently in all cases hereby delivering an excellent basis for the in depth zoogeographic analysis in chapter 4 . As members of the genus Zygaena represent complicated species groups in several cases, one of the most complicated ones is presented in the short chapter 3, the Z . purpuralis/minos complex. Chapter 4 - with almost 200 pages the largest of this volume and maybe its core - is addressing the distribution and zoogeography of Zygaena. It provides detailed information about the distribution (geographic range and altitudinal distribution) for all species and is delivering in depth analysis on the distribution dynamics and its drivers, but also details on the zoogeography of each of 15 distinguished geographic entities. Everyone interested in biogeography in general will be fascinated by the profound knowledge found in this chapter and the very well elaborated analyses and conclusions. There is only few group of organisms understood in so much detail and presented with so much background knowledge!

The fifth chapter is giving a lot of information on the morphology and behavioural traits of all stages illustrating everything with excellent photographs and drawings. As always in this book, the number, quality and didactic excellence of all figures is impressive. Chapter 6 is addressing another fascinating aspect for which the genus Zygaena is particularly interesting: The variation of phenotypes. Based on many examples, the authors show the enormous morphological diversity in particular of the colour patterns within burnet moth species, their geographic diversity, but also the regional parallelisms in pattern evolution. By discussing these examples based on imagoes and preimaginal stages, the authors provide general conceptional ideas for these and related phenomena. The following chapter is going more into depth here, dealing with many detailed examples of the regional morphologic diversity within species. In particular, the phenomena of the regional melanisms and spot reniformations are described and discussed in much detail.

The eight and last chapter of volume 1 is going into the history of Zygaena research. It is presenting the important personalities working with Zygaena starting with Eleazar Albin (1690-1742) highlighting the contributions to Zygaena research of all of them. This chapter is enriched by portraits and photographs of these entomologists as well as some replica of plates depicting burnet moths from the books they have produced. Following this, details on the so far 14 International Symposia on Zygaenidae are presented also unravelling the items discussed and the existing scientific networks. The book is closed by excellent photographs of male and female genitalia of all Zygaena species with their respective subspecies, a full list of references and a glossary giving important definitions.

Last but not least, this book is not only packed with an incredible amount of information, but it is also one of the most beautiful and impressive books I have had in my hands over the last years. Therefore, this book is a great treasure for all interested in the natural history of insects and for everyone simply loving such wonderful books. There would be so much more to write on this work that would burst such a short review. I therefore simply recommend reading and enjoying this spectacular result of science! 